\title{
Strategic and Tactical Problems in Fintech and E-business Companies
}

\author{
Assoc. Prof. Dr. Pavel Petrov \\ University of Economics - Varna, Varna, Bulgaria \\ petrov@ue-varna.bg \\ Dr. Nikolay Valov \\ University of Ruse "A. Kanchev", Ruse, Bulgaria \\ npvalov@uni-ruse.bg
}

\begin{abstract}
The activities of fintech companies depend entirely on information technologies. In the case of a startup company, there are several stages of acquiring and using of information technologies for the needs of the business. In relatively small fintech companies, intuitive decision-making by managers regarding information technologies is in most cases enough for normal functioning of the business. But in relatively large fintech companies with complex organizational structures, it is very likely to emerge the so called "bottlenecks" - in them, the execution of some processes is delayed due to insufficient processing and handling capacity. This publication discusses some problems in fintech and e-business companies concerning the strategic and tactical management levels.
\end{abstract}

Keywords: fintech companies, e-business companies, strategy, information system, information technologies.

JEL Code: G20; doi:10.36997/IJUSV-ESS/2019.8.3.55

\section{Въведение}

Дейността на финтех компаниите зависи изцяло от информационните технологии. В случай на стартьп се преминава през няколко етапа на придобиване и използване на информационни технологии за нуждите на дейността. През пьрвия етап основно се закупува хардуер (изчислителна, комуникационна и др. техника) и софтуер (програмно осигуряване системен, приложен, информационни системи за управление и др.). През втория етап протича процес на поддържане на използвания хардуер и софтуер. На трети етап се появява нуждата от обновяване на използваните информационни технологии. Последните два етапа са с най-голяма продължителност в живота на компанията.

При сравнително малки финтех компании интуитивното вземане на решения от мениджърите по отношение на информационните технологии в повечето случаи е достатъчно за нормалното функциониране.

При сравнително големи финтех компании, със сложна организационна структура е твърде вероятно да започват да се появяват т.нар. "тесни места" - в тях изпълнението на някои процеси се задържа поради недостатъчен капацитет за обработка и справяне с проблема. Това оказва съществено влияние върху процесите, които технологично са след "тесните места", където най-често се появяват проблемите, тъй като се загубва синхронността при съвместната работа на отделните структурни звена. Появява се ситуация, при която служителите и мениджърите на ниски и средни нива на управление са неудовлетворени от работата си, въпреки наличието на съвременни информационни технологии. Възникват редица въпроси - например как ще се развива информационната система (Radev\&Aleksandrova, 2017) и има ли смисъл да се инвестира в ново появяващи се технологии. Поради подобна неопределеност, мениджърите и служителите във финтех компанията може да не могат да осмислят достатъчно добре целите, задачите, принципите на функциониране и поради това да не могат да оптимизират всички аспекти от дейността си.

Появата на подобен проблем - разминаване на извършваната дейност с бизнес стратегията на финтех компанията, може да доведе до увеличаване на рисковете по всички направления: от една страна, при оперативната дейност - неспособност за справяне със заплахи към информационната сигурност - дефектиране на техника, хакерски атаки, вируси, кражба на данни и пр. От друга страна тези рискове може да са от тактическо естество - при 
анализа, проектирането, документирането, закупуването и внедряването на информационни технологии. Възможно е да не се избере оптимално решение по отношение на информационните технологии, да не се използват напълно потенциалните възможности на внедрените информационни технологии, да има несъответствия между изградената инфраструктура и насоките на развитие на информационните технологии, да има организационни и технически грешки при инсталирането на различни информационни системи, да не може да се поддържа и развива информационната система на приемливо ниво, да няма предвидени мероприятия при извънредни ситуации, да не може инфраструктурата да издържа нормални или пикови натоварвания, да не се прилагат оптимални процедури по техническа поддръжка и др.

Намаляването на риска от подобни заплахи, които възпрепятстват нормалната дейност, а от там и постигането на стратегическите бизнес цели, е възможно да се постигне чрез създаването във формален явен и писмен вид на Стратегия за развитие на информационните технологии, която да дефинира дългосрочните цели и "посоката на движение" в областта на информационните технологии. Стратегията за развитие на информационните технологии би следвало да е част от бизнес стратегията и да усилва бизнес предимствата на финтех компанията спрямо конкурентите. Нейното прилагане би трябвало да способства за успешното съществуване и развитие на компанията, като конкурентноспособен субект в една силно динамична пазарна среда. В този смисъл, можем да обобщим, че докато бизнес стратегията определя какво е необходимо да прави компанията, то Стратегията за развитие на информационните технологии отговаря за това как от гледна точка на информационните технологии би следвало да се реализира бизнес стратегията.

\section{1. Стратегически и тактически проблеми при финтех компаниите}

При разработването на Стратегия за развитие на информационните технологии е необходимо да се отчита степента на важност на информационните технологии за отделните бизнес дейности и да се осигури висока степен на непрекъсната работа на информационната инфраструктура и информационните услуги, тъй като при финтех компаниите те са от високо значение за основните бизнес процеси - електронна търговия, онлайн банкиране, интернет услуги и др. Като част от общата стратегия на компанията, Стратегията за развитие на информационните технологии е необходимо да указва по какъв начин на базата на актуални към момента информационни технологии могат да се подобрят бизнес процесите с цел увеличаване на печалбата - намаляване на разходите или увеличаване на приходите, и придобиване на конкурентни предимства. В идеалния случай задачата на Стратегията за развитие на информационните технологии е да намалява оперативните разходи за информационната инфраструктура, като за сметка на тях увеличава тези за внедряването на нови технологии за подкрепа на бизнес дейностите.

При големи финтех компании е удачно с цел по-голяма ефективност на разработената Стратегия за развитие на информационните технологии, предварително да се разработят отделни стратегии по основните дейности на компанията, след което Стратегията за развитие на информационните технологии е необходимо да ги осигурява и оптимизира. По този начин Стратегията за развитие на информационните технологии се съобразява с дългосрочните и средносрочните потребности на бизнеса, което позволява да се повиши ефективността на служителите, а от там и на компанията като цяло, по отношение на вземане на решения, работа с доставчици и клиенти, съвместна работа вътре в компанията и др.

При създаване на продукт или услуга от финтех компаниите не е подходящо създаването на интегрирани, с висока степен на сложност (Aleksandrova, 2018), "монолитни" информационни системи, в които се прави опит да се обхванат всички бизнес процеси, тъй като времето за изграждане на подобни системи е много голямо. В периода от време между проектирането на продукта или услугата и нейното внедряване могат да се сменят не само хардуерните, софтуерните и комуникационните средства, които се използват при 
изграждането на информационната система, но и бизнес процесите - начините на управление, характерът на дейността и т.н., във финтех компанията. Това би наложило постоянен реинженеринг на продукта или услугата, което би увеличило значително разходите за разработка и поддръжка. Поради тези причини, модулното изграждане на информационната система, която стои зад продукта или услугата на финтех компанията е поефективен подход. Това е обосновано и с оглед на възприемането, че това са организации с относително по-кратьк "живот", но за сметка на това "по-бурен". В идеалния случай финтех компанията би следвало да използва в комбинация няколко уеб базирани продукта от тип свободен софтуер с лиценз, съвместим с GNU GPL.

Използването на готови стандартни решения от тип свободен софтуер за реализацията на продукта или услугата на финтех компанията може значително да опрости първоначалните етапи при изграждането на финтех продукта или услугата, а и в последствие да носи по-големи ползи и предимства за бизнеса. Някои от ползите при използването на готови стандартни решения от тип свободен софтуер са следните: намалява се зависимостта от доставчици на софтуер и хардуер; управлението на организацията на работа при нужда може лесно да се променя чрез смяна и доработка на различни софтуерни модули, тъй като изходния код е наличен и софтуерния лиценз позволява това; на външни партньори може относително лесно да се предостави достьп до информация за състоянието на проектите, по които се работи; лесен обмен на данни между различни приложения, благодарение на използването на общоприети стандарти.

Допълнителни предимства при използването на готови стандартни решения от тип свободен софтуер е, че: технологиите, на които се базира голяма част от свободния софтуер, са открити и се базират на документирани, стандартизирани, общо- и леснодостьпни протоколи и файлови формати; минимални разходи при изграждане и обновяване, тъй като по-голямата част от свободния софтуер е безплатен и освен с изходния си код, се разпространява и с правото да се използва, копира, модифицира и разпространява без да се иска разрешение от носителя на авторското право; висока сигурност и отказоустойчивост, тъй като се използва софтуер, програмни библиотеки и технологии, които са се доказали в практиката.

\section{2. Стратегически базови модели за взаимодействие при дигитализация на}

\section{бизнеса}

В основата на дигитализацията стои стремежът за преминаване към нови и съвременни форми за организация на бизнеса. Когато за извършване на бизнес процесите преобладаващо се използват електронни средства, то тази организация е известна като "електронен бизнес". Електронният бизнес е форма на организация на бизнеса, при която бизнес процесите, обмяната на информация, финансовите транзакции и прочие дейности се автоматизират чрез информационни системи, които в последните години използват интернет технологии като основна комуникационна среда. Тази среда се използва и за предлагане на различни услуги на мобилни устройства - таблети и смартфони. Терминът за пръв път е използван от Луи Герстнер от IBM през 1996 година (Amor, 1999). Постепенно преминаването към тази форма на организация на бизнеса става основна цел на редица компании и банки, появяват се и нов вид финансови компании, наричани с общото наименование "финтех". Дигитализацията на финансовите услуги води до много нови онлайн услуги и по-лесен достьп до банковия пазар за клиентите. При прилагане на електронен бизнес се появяват възможности за разширяване на базата от клиенти, разширяване на географския обхват, прилагане на електронен маркетинг и др.

Едновременно с новите възможности, които предлага електронния бизнес, се появяват и нови технологични рискове - проблеми със софтуера, базите от данни, идентификацията на потребителите, електронния подпис, конфиденциалността на информацията и др. Преминаването на една фирма към електронен бизнес може да наложи промени в организационната структура и начина на осъществяване на връзка с бизнес партньорите, 
което от своя страна да окаже влияние върху ролята и задълженията на служителите и мениджърите на различните управленски нива. За минимизиране на рисковете от прилагане на нови информационни технологии е необходимо да се поддържа подходяща информационна инфраструктура (Panayotova et al., 2016) и да се използва система за управление на информационните технологии.

В зависимост от субектите, информационните потоци и услугата, която е предмет на електронен бизнес, се забелязват няколко устойчиви модела на електронно бизнес взаимодействие (Bjørner, 2003; Adam, 2009), представени в таблица 1.

Таблица 1. Основни модели за електронно бизнес взаимодействие.

\begin{tabular}{|l|c|c|c|c|}
\hline & Бизнес & Потребител & Служител & Дьржава \\
\hline Бизнес & В2B & B2C & B2E & B2G \\
\hline Потребител & C2B & C2C & - & C2G \\
\hline Служител & E2B & - & E2E & - \\
\hline Дьржава & G2B & G2C & G2E & G2G \\
\hline
\end{tabular}

Основните характеристики на тези модели са следните:

- B2B (Business to Business). Компании, обикновено във вертикална интеграция, реализират продажби по веригата на снабдяване. Основни задачи са: осигуряване на висока степен на синхронизация между субектите чрез предоставяне на нужната информация навреме, повишаване на нивото на сигурност при обмена на информация. Необходимо е предварително определяне на стандарти при обмен на данни.

- B2C (Business to Consumer). Реализиране на продажби на стоки и услуги на крайни потребители. Основни задачи са: осигуряване на високо качество на съдържанието, удобство, сигурност. Възможни са редица проблеми от правен характер.

- B2E (Business to Employee). Автоматизиране на управленските функции на мениджърите чрез обмяна на съобщения вътре в компанията (ел. поща, аудио и видео връзка и др.), организация на работата (графици, списъци със задачи и др. ). Прилагат се и Системи за управление на знанията, които се използват за обучение на нови служители или за усъвършенстване на уменията.

- B2G (Business to Government). Електронно подаване на фирмени данъчни, митнически и друг вид декларации и информация до държавни и общински органи.

- C2B (Consumer to Business). Потребител (може и бизнес потребител) публикува поръчка за стока или услуга и максимална цена, след което в рамките на определен период от време различни компании кандидатстват да изпълнят проекта. Потребителят разглежда заявките и избира компания, която да изпълни поръчката.

- C2C (Consumer to Consumer) - Потребители се самоорганизират на базата на общи интереси или проблеми. Дава се възможност да се споделят мнения, съвети, полезен опит, да се правят сравнения по определена стока или услуга с цел повишаване на качеството й. Понякога потребителите се организират за закупуването на определена стока по цени "на едро" от определен производител или търговец, като ползват отстъпка на база заявено поголямо количество от един продукт. Процесите е необходимо да се следят от специалисти, за да се минимизира влиянието от разпространяването на погрешна информация. Съществуват специализирани сайтове за безплатни обяви, аукциони и форуми, където потребителите могат да извършват онлайн плащания към други потребители (например PayPal). За един от най-големите сайтове-аукциони се счита еВау.com, които започва своята работа през 1995 г.

- C2G (Consumer to Government). Подаване на електронни данъчни декларации и онлайн ползване на услуги предлагани от държавните органи.

- E2E (Employee to Employee). Обмяната на информация между служителите по електронен път, с цел да се минимизира хартиения документооборот. Целта е да се автоматизират процесите на намиране на нужната информация, обработване и предаване 
между отделните служители, като се намалява ръчната обработка, пести се време и се повишава качеството на работа.

- G2B (Government to Business). Предлагане на нормативна и друга информация, необходима за функционирането на бизнеса. Например: Държавен вестник, списък с фирми регистрирани по ДДС, условия за кандидатстване по проекти и т.н.

- G2C (Government to Consumer). Информиране на гражданите по въпроси, които са свързани с взаимоотношенията им с държавата - неплатени данъци, глоби, издирване, съдебни решения, прием в детски градини, издаване на лични документи, регистрация на превозни средства и др.

- G2E (Government to Employee). Подобно на B2E, но с отчитане на спецификата на работа на държавната администрация. За реализиране на бизнесмоделите Е2Е, В2E и G2E могат да се използват софтуерни продукт от тип групеьр, чрез които да се управлява работния процес, като се дават на съответните служители необходимите данни на определен етап от дейността. Подобни системи са особено подходящи за използване при добре структурирани процеси, където е възможно точно да се дефинират правила на всяка стъпка от работния процес.

- G2G (Government to Government). Подобряване на работата на държавните органи в една или различни държави чрез увеличаване на нивото на координираност между тях, чрез по-ефективно споделяне на информация и координиране на действията. Много често целта е откриване и предотвратяване на митнически и данъчни злоупотреби, борба с организирана и криминална престьпност, ускоряване работата по съвместни проекти и др.

От гореизброените основни модели могат да се образуват и редица производни модели за конкретни ситуации, като се заменят по подходящ начин субектите от моделите. Например, категориите Бизнес - Потребител - Служител могат да се заменят съответно с категориите, Учебно заведение - Обучаем - Преподавател, Лечебно заведение - Пациент Лекар, Застраховател - Застрахован - Застрахователен агент и др., като общото между тях е, че първият субект като юридическо лице предлага продукт или услуга, вторият като частно лице я потребява, а третият е физическия извършител.

Тези електронни бизнесмодели се реализират под формата на информационни системи, използващи главно уеб технологиите (Panayotova, 2015). В зависимост от нивото на взаимодействие, което предлагат използваните уеб технологии в бизнеса, могат да се разграничат няколко т.нар. "поколения":

- Първо поколение. Уеб сайтът представлява подобие на информационно табло, брошура или витрина. Информацията се предава еднопосочно и потребителя не взаимодейства със сайта, а само пасивно възприема информацията, като се придвижва между страниците.

- Второ поколение. Уеб сайтьт предлага възможност за взаимодействие, чрез система за тьрсене на определена информация за стока или услуга. Има двустранен обмен на информация под формата на заявка - отговор.

- Трето поколение. Уеб сайтът предлага възможност за бизнес взаимодействие например потребителят може да поръча стоки или услуги и да заплаща онлайн. На сайта има връзки към транспортни фирми, специалисти и други компании, с които компанията има договори.

- Четвърто поколение. Уеб сайтът е интегриран в бизнес процесите на компанията и е основно средство за връзка с клиенти и бизнес партньори. Например, поръчката на стока автоматично води до действия по преместването й от склада към отдела за доставка и счетоводното отчитане на операцията.

В последните години голямо развитие се забелязва при системите за електронен бизнес в сферата на тьрговията, банковото дело, транспорта (например самолетните превози) и туризма (например резервации). Като основна среда за бизнеса убедително се налагат уеб технологиите, които имат следните по-съществени предимства:

- Устойчивост. Програмните средства, които се използват са изпитани в много 
натоварена среда - Интернет, в продължение на години. Това гарантира надеждност на работата (Petrova et al., 2019) и мащабируемост при нарастване на интензивността на работа (Dimitrov et al., 2017) с документите;

- Удобен потребителски интерфейс. Интерфейсните средства, които се използват, са същите, с които потребителите започват да работят с Интернет (много често и у дома) и са интуитивно разбираеми. Премахва се или се намалява нуждата от обучение и преквалификация на служителите за работа с хипертекстови документи;

- Универсалност. Използването на стандартизирани приложни програмни интерфейси осигурява независимост от компютьрни платформи (например десктоп и смартфони) и операционни системи (например Windows и Android).

\section{3. Тактически проблеми при дигитализация на бизнеса, отнасящи се за информационните системи}

Динамиката на бизнес процесите в икономиката налагат създаването на информационни системи с по-голяма степен на оперативност. Съответно използваните информационни технологии (Balabanova et al., 2018) е необходимо да осигурят възможност за съхраняване, обработване и представяне на информация в реално време. Това е възможно да се реализира чрез използването на Интернет, съвременни програмни и комуникационни средства. Изграждането им, от друга страна, се определя от различни специфични изисквания и ограничения, като: изискване за работа в реално време или изискване за работа в режим "онлайн", определено ниво на производителност, сигурност, безотказност, възможност за бъдещо развитие и надграждане на функционалността, мащабируемост, настройване, стандартизация, време за разработка, цена на разработка, цена на поддръжка и T.H.

Съществен момент при разработката на информационна система е определяне на това с какви средства да се реализира потребителския интерфейс. Към настоящия момент на развитие на информационните технологии разработката на потребителския интерфейс (Georgiev et al., 2018) на една информационна система се свежда до следните основни варианти:

- Десктоп приложение, използващо прозоречен интерфейс и работещо под графична операционна система от типа Windows, X Windows, Mac OS и други подобни;

- Уеб базирано приложение, работещо посредством използването на уеб браузър, който се свързва със сървърната част на разпределено клиент-сървър уеб приложение;

- Мобилно приложение, специално предназначено за използване на смартфон или таблет, работещо под операционна система Android, iOS и други подобни.

От тези варианти най-универсален е подходът за разработка на информационна система с уеб базиран интерфейс, тъй като тя може да се използва от широк кръг устройства - както от настолни и преносими персонални компютри, така и от мобилни устройства смартфони и таблети. Докато мобилните системи и десктоп системите могат да се използват само на определен тип хардуерни устройства със съответна специфична операционна система, то уеб технологиите (Tonkova\&Stoev, 2007) са независими от вида на устройството и операционната система, работеща на заден план.

\section{Заключение}

Дигитализацията на бизнеса води до много нови възможности, но едновременно с това, се появяват и нови технологични рискове. Прехода към дигитализация на бизнеса може да наложи промени в организационната структура. Информационните технологии са в основата на дейността на финтех и е-бизнес компаниите. По време на функционирането им, възникват различни видове проблеми и заплахи, свързани с използването и развитието на информационните технологии. Намаляването на подобни рискове може да се чрез създаването на Стратегия за развитие на информационните технологии, която да дефинира дългосрочните цели в областта на информационните технологии. В тактически план, 
използването на готови стандартни решения от тип свободен софтуер за реализацията на продукта или услугата на финтех компанията може значително да опрости пьрвоначалните етапи при изграждането на финтех продукта или услугата, а и в последствие да носи поголеми ползи и предимства за бизнеса.

Преминаването на една фирма към е-бизнес може да наложи промени в организационната структура и начина на осъществяване на връзка с бизнес партньорите, което от своя страна да окаже влияние върху ролята и задълженията на служителите и мениджърите на различните управленски нива. В зависимост от субектите, информационните потоци и услугата, която е предмет на е-бизнес, се забелязват няколко устойчиви модела на електронно бизнес взаимодействие. Всеки от моделите има свои собствени специфични характеристики. Като основна среда за бизнеса убедително се налагат уеб технологиите, които съществени предимства. Могат да се разграничат няколко поколения уеб технологии в бизнеса, в зависимост от нивото на взаимодействие, което предлагат. Уеб базираните информационни системи могат да се използва от широк кръг устройства - както от настолни и преносими персонални компютри, така и от мобилни устройства - смартфони и таблети.

\section{References}

1. Adam, C. (2009). Considerations on Terminology and Concepts Used in the Electronic Commerce Literature. Marketing From Information to Decision, (2). pp.29-42.

2. Aleksandrova, Y. (2018). Application of Machine Learning for Churn Prediction Based on Transactional Data (RFM Analysis). 18 International Multidisciplinary Scientific Geoconference SGEM 2018: Conference Proceedings, 18(2.1), pp.125-132.

3. Amor, D. (1999). The e-business (r)evolution. Upper Saddle River. Prentice Hall.

4. Balabanova I., Georgiev G., \& Sadinov, S. (2018) Synthesizing of Models for Identification of Teletraffic Markov Chains by Artificial Neural networks and Decision Tree Method. Journal of Electrical Engineering, vol.5, Slovakia, pp.379-384.

5. Bjørner, D. (2003). Domain Models of "The Market" - In Preparation for E-Commerce. Practical Foundations of Business and System Specifications. Kluwer.

6. Dimitrov, G.P., Panayotova, G. \& Kostadinova, I. (2017). Approaches for optimization of web pages loading via analysis of the speed of requests to the database. 4th International Conference on Artificial Intelligence and Pattern Recognition, AIPR 2017, pp.58-63.

7. Georgiev G., Balabanova I., Kogias P., Sadinov S., \& Kostadinova, S. (2018). Identification of Sine, Squire, Triangle and Sawtooth Waveforms with Uniform White and Inverse F Noises by Adaptive Neuro - Fuzzy Interface System. Journal of Engineering Science and Technology Review, 11(3), pp.128-132.

8. Panayotova, G., Dimitrov, G., Petrov, P. \& Bychkov, O. (2016). Modeling and data processing of information systems. 3rd International Conference on Artificial Intelligence and Pattern Recognition (AIPR), Lodz, Poland, IEEE, pp.154-158.

9. Panayotova, G.S. \& Dimitrov, G.P. (2015). Design of web-based information system for optimization of portfolio. Proceedings of the 13th International Symposium on Operational Research, SOR 2015, pp.193-198.

10. Petrova, S., Stefanov, S., Ivanov, S., Sergeev, A. \& Getova, I. (2019). Information Systems Used in Bulgarian University Libraries as Online Public Access Catalogs. 19 International Multidisciplinary Scientific Geoconference SGEM 2019. 19(2.1), pp.353-360.

11. Radev, M., Aleksandrova, Y. (2017). A New Approach For The Education Process In Bulgarian Universities. The Second Conference on Innovative Teaching Methods (ITM 2017), Varna, pp.216-219.

12. Tonkova, E. \& Stoev, S. (2007). Blogs, Web Discussion Forums and their Business and Consumer Implications. Innovation Entrepreneurship and Competitiveness: In the Balkan and Black Sea Contries: Intern. Conf., Kavala, Greece. 